\title{
Precise instructions determine participants' memory search strategy in judgments of relative order in short lists
}

\author{
Michelle Chan \\ University of Alberta, Edmonton, Alberta, Canada \\ BERNhaRd Ross AND GUY EARLe \\ Rotman Research Institute, Baycrest, Toronto, Ontario, Canada \\ AND \\ JeREMY B. CAPLAN \\ University of Alberta, Edmonton, Alberta, Canada
}

\begin{abstract}
Memory often requires knowledge of the order of events. Previous findings about immediate judgments of relative order in short, subspan lists are variable regarding whether participants' strategy is to search memory in the forward direction, starting from the first list item and progressing toward the end item, or in the backward direction, starting from the end item and progressing toward the start. We asked whether wording of the instructions influences participants' search direction. Participants studied sequences of three to six consonants, and for an immediate, two-item probe of each list, judged which probe was presented earlier ("earlier" instruction) or later ("later" instruction) on the list. Forward and backward searches were supported for "earlier" and "later" instructions, respectively. Our findings suggest that participants have more than one effective strategy for order judgments in short lists, and that subtle instructional differences can bias memory search in either the forward or backward direction.
\end{abstract}

Memory researchers have striven to explain memory for order separately from memory for items and associations (Murdock, 1974). Memory for order is ubiquitous; even in free recall, for which order is not required, participants still preserve some presentation-order information in their recall sequences (Kahana, 1996), suggesting that order cuing is a pervasive characteristic of human memory, as argued systematically by McDaniel and Bugg (2008). A direct way to test memory for order is to present the participant with a two-item probe and ask which probe item came later in the list, usually called "judgment of relative recency" (JOR). JOR itself has broad relevance to everyday experience. For example, two dishes are ready and the waiter needs to determine their relative order to deliver them to their respective diners. The JOR paradigm has been extensively studied with long (supraspan) lists (e.g., Fozard, 1970; Wolff, 1966; Yntema \& Trask, 1963; see McDaniel \& Bugg, 2008, for a review), but, surprisingly, much less is known about relative order judgments in short (subspan) lists. In the few studies that have attempted to elucidate the memory-search process underlying judgment of relative order in short lists, there are some consistent findings (Table 1). For example, an important distinction in memory search models is whether the memory com- parison is exhaustive or self-terminating. In exhaustive search, the memory comparison process continues regardless of a positive or negative match. In self-terminating search, the memory comparison process ends with a positive match. A significant effect of serial position on response time is a clear-cut sign of self-terminating search (Townsend \& Wenger, 2004), and all studies of short-list JORs have reported significant serial position effects. However, the direction of the self-terminating search has been inconsistent across studies (Table 1), with some supporting a backward search strategy, wherein participants start at the end of the list and search toward the start, and one supporting forward search, wherein participants start at the first list item and progress toward the end of the list. Noting that the instructions differed across studies, we speculated that subtle differences in instructions may influence a participant's choice of strategy by directing attention predominantly to the start or the end of the list. Our aim was to test this hypothesis by manipulating the wording of instructions between groups of participants.

In Figures 1A and 1B, we illustrate the hypothetical response time (RT) as a function of the serial positions of two probe items in the study list for six-item lists to show how serial position effects can be used to determine

M.Chan,mc3@ualberta.ca 
Table 1

Summary of Four Short-List Judgment of Relative Order Studies

\begin{tabular}{lllll}
\hline & $\begin{array}{c}\text { Sternberg (1969) } \\
\text { Experiment 8 }\end{array}$ & Muter (1979) & Hacker (1980) & $\begin{array}{c}\text { McElree \& } \\
\text { Dosher (1993) }\end{array}$ \\
\hline Material type & Digits & Nouns & Consonants & Consonants \\
List length & $3-6$ & 4 or 10 & 9,11, or 13 & 6 \\
Number of list presentations & 2 & 1 & 1 & 1 \\
Interstimulus interval & Not reported & $25 \mathrm{msec}$ & $100 \mathrm{msec}$ & $50 \mathrm{msec}$ \\
Retention interval & $1,000 \mathrm{msec}$ & $1,000 \mathrm{msec}$ & $100 \mathrm{msec}$ & $550 \mathrm{msec}$ \\
Forward recall attempt & Yes & No & No & No \\
Lags probed & Successive serial & All combinations & All combinations & All combinations \\
& positions only & of list positions & of list positions & of list positions \\
Scanning direction & Forward & Backward & Backward & Backward \\
\hline
\end{tabular}

Note-Instructions for Sternberg (1969, Experiment 8, p. 452): “. . decide whether the left-to-right order of the pair was the same as its temporal order in the list, or reversed.” Instructions for Muter (1979, p. 163): “. . . press Key 1,2, or 3 if the top test item had been presented more recently in the list, and Key 4, 5, or 6 if the bottom test item had been presented more recently in the list." (Keys indicate level of confidence.) Instructions for Hacker (1980, p. 653): “. . . identify the more recent of the two items.” Instructions for McElree and Dosher (1993, p. 296): "[Indicate] which of the left-right test items was judged more recent."

search direction. We assumed that error rates are equivalent across probe types; RT is therefore the sensitive dependent variable, which is usually the case for subspan lists (Table 1). Because probe items have to be different, one probe item will always come from an earlier list position than the other. Thus, we plotted RT as a function of two independent variables: the serial positions of the earlier- and the later-probe item. Figure 1A illustrates the hypothetical RT if search were in the forward direction and self-terminating. In a forward search, participants compare the probe items with studied items, starting from the first studied item and progressing toward the end of the list. In a self-terminating search, the search terminates once a match to one of the probe items has been found. Therefore, we predict that RT will increase with increasing serial position of the earlier item. For correct responses, which are considered here exclusively, the earlier-probe item will be found before the later one has been reached, and RT will be independent of the serial position of the later-probe item. Accordingly, in addition to RT's increasing with earlier-probe item position for any given position of the later item, RT is invariant with position of the laterprobe item for any given position of the earlier item. The 3-D bar graph pattern can be approximated by two regression lines, one describing the change in RT as a function of the serial position of the earlier-probe item in the study list, the other describing the change in RT as a function of the position of the later-probe item (each while holding the position of the other probe item constant). Our prediction is that the case of a self-terminating forward search will be characterized by a positive slope of the regression line of RT versus position of the earlier-probe item (Figure $1 \mathrm{C}$ ) and by a flat function of RT versus position of the later-probe item (near-zero slope, Figure 1D). We apply the same logic to predict the dependence of RT on the two probe items' serial positions for the backward self-terminating search, wherein participants start from the end of the list and progress toward the start. RT will increase with decreasing serial position of the later-probe item. Self-terminating backward search will lead the participant to encounter the later-probe item first, and RT will not depend on the position of the earlier-probe item (Figure 1B). This strategy will be indicated by a negative slope of RT versus position of the later-probe item (Figure $1 \mathrm{~F}$ ) and a near-zero slope of RT versus position of the earlier-probe item (Figure 1E).

\section{Serial Versus Parallel Processing}

Modelers also distinguish between serial and parallel search strategies, but serial and parallel models can mimic each other (e.g., Townsend \& Wenger, 2004). In serial search, list items are scanned one after the other, producing a linear relationship between RT and list length (LL), such that each additional item increases the RT by a fixed amount. In parallel search, all items are evaluated simultaneously, producing no change of RT with LL. A variety of parallel models incorporate the notion of "limited capacity," wherein the presence of additional items in the search set slows all search rates, enabling parallel models to produce an approximately linear increase in RT with LL (Murdock, 1982). Thus, serial and parallel models can make many of the same predictions (Townsend \& Wenger, 2004). Because parallel models are equally plausible, it is important to understand how the interpretation might change in a parallel search model. The parallel-model analogue of forward and backward search predictions is understood by replacing the notion of scanning direction with forward- and backwardbiased activation gradients, such that items will be searched faster or slower as a function of position even if all comparisons proceed in parallel. We will consider a parallel-model interpretation of the results in the Discussion.

\section{The Present Experiment}

To test the hypothesis that instructions could bias search direction even if the logical judgment is the same, we manipulated the instructions between two participant groups. One group received the "later" instruction, paralleling Hacker (1980), McElree and Dosher (1993), and Muter's (1979) experiments. The other group received the "earlier" instruction, used by Kesner, Hopkins, and Fineman (1994), asking participants which of two probe stimuli occurred earlier in a studied list. 
A

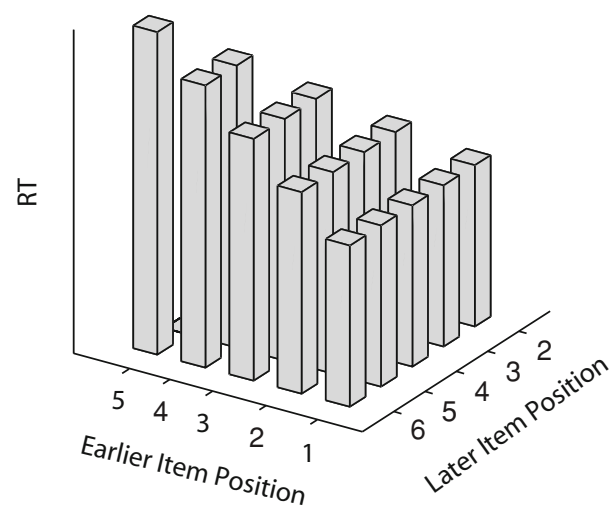

C Serial Position Slope for the Earlier Item

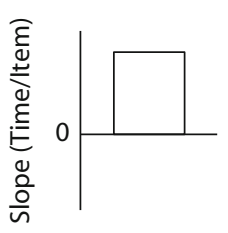

G

Earlier Instruction (Data)



I Serial Position Slope for the Earlier Item

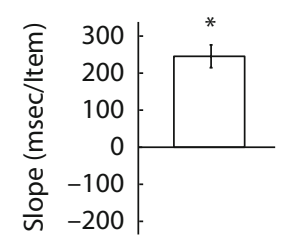

D Serial Position Slope for the Later Item

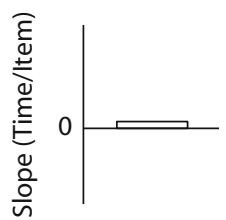

B Backward Self-Terminating (Schematic)

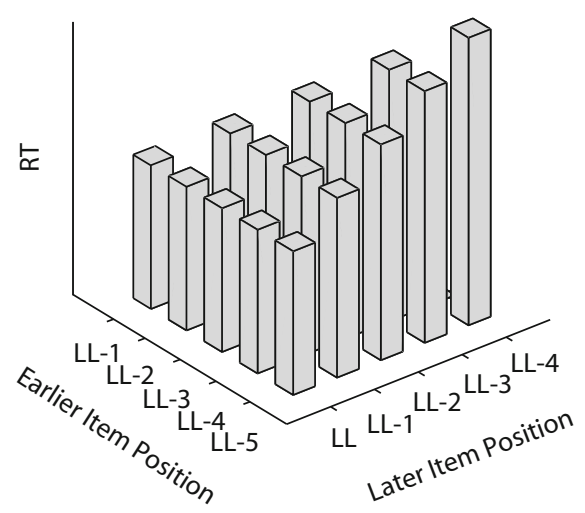

E Serial Position Slope for the Earlier Item

F Serial Position Slope for the Later Item

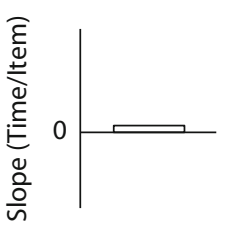

H

H Later Instruction (Data)

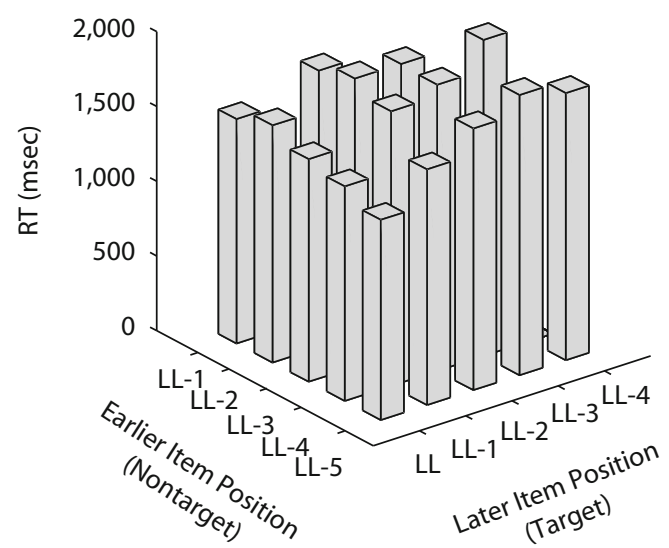

K Serial Position Slope for the Earlier Item

L Serial Position Slope for the Later Item

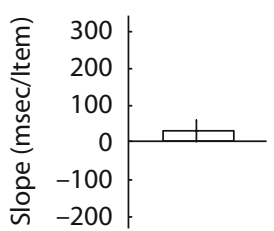

Figure 1. Hypothetical response times (RTs; arbitrary units) for forward (A, C, D) and backward (B, E, F) self-terminating search. RT is plotted as a function of the positions of the earlier- and later-probe items in the study list (A, B). Note that for forward scanning (A), serial position is relative to the start of the list (Position 1), whereas for backward scanning (B), serial position is measured relative to the end of the list (position equal to the list length, abbreviated LL). Observed RT is plotted for "earlier" (G, I, J) and "later" $(H, K, L)$ instructions. Slopes of the earlier-probe item position (while holding the later-probe item position fixed) are summarized in the single bar graphs in panels $\mathrm{C}$ and $\mathrm{E}$ (hypothetical data) and $\mathrm{I}$ and $\mathrm{K}$ (observed data). Likewise, slopes of the later-probe item position (while holding the earlier-probe item position fixed) are summarized in the single bar graphs in panels $D$ and $F$ (hypothetical data) and $J$ and $L$ (observed data). Error bars in slope bar graphs denote standard error of the mean. Asterisks denote significantly nonzero slopes $(p<.05)$. 


\section{METHOD}

\section{Participants}

Eighty-eight University of Alberta undergraduate students with normal or corrected-to-normal vision participated for partial course credit. Data from 8 participants were excluded because their error rates were high $(>30 \%)$. In each group, 40 participants were included in the final analyses.

\section{Materials}

Stimuli were 16 consonants from the English alphabet displayed in capital letters. The letters $S, W, X$, and $Z$ were excluded for consistency with another study (van Vugt, Schulze-Bonhage, Litt, Brandt, \& Kahana, 2009). Each list comprised 3-6 consonants drawn at random without replacement from the stimulus pool, with the restriction that they must not have appeared in the two preceding lists. Probability was equal for each consonant/serial-position combination. LL was randomly drawn from a uniform distribution from three to six items. The experiment was created using the Python ExperimentProgramming Library (Geller, Schleifer, Sederberg, Jacobs, \& Kahana, 2007) and modified from an experiment by van Vugt et al. Probes were pairs of items drawn from the just-presented list and were sampled without replacement for a given list length from the set of all possible probe position permutations until all permutations were used; then the unique probe position pairs were used, after which the permutation set was restored as many times as needed. The use of all probe permutations is in line with Hacker (1980), McElree and Dosher (1993), and Muter (1979) and is standard for long-list JOR studies (e.g., Yntema \& Trask, 1963) but deviates from Sternberg's (1969) procedure, which tested only nearest-neighbor positions.

\section{Procedure}

The time course of a trial is depicted in Figure 2. After an orienting stimulus, list items were presented for $575 \mathrm{msec}$ each, with an interstimulus interval of $175 \mathrm{msec}$. Following the last list item, there was a 2,500-msec retention interval (to avoid the kinds of recency effects that have previously confounded interpretation of a related item-recognition paradigm; see Jensen \& Lisman, 1998), after which a two-item probe was displayed. Participants responded with the "." and "/" keys and were instructed to respond as quickly as possible without compromising accuracy. After a posttrial delay of $500 \mathrm{msec}$, the participant pressed any key to continue. The next trial began after a delay of $400 \mathrm{msec}$. The "earlier" instruction (Figure 2A) was as follows:

Judge which of the two letters came earlier on the list you just studied. Press the "/" key if the earlier item from the list is presented on the right side of the screen and the "." key if the earlier item is on the left side of the screen.

\section{The "later" instruction (Figure 2B) was to}

Judge which of the two letters came later on the list you just studied. Press the "/" key if the later item from the list is presented on the right side of the screen and the "." key if the later item is on the left side of the screen.

Keypresses were logged for both accuracy and RT. Each session comprised one block of 8 practice trials, followed by nine blocks of 20 experimental trials, for a total of 188 trials. In the practice block, immediate feedback on correctness was given after each trial. The experimenter remained in the testing room during the practice block to ensure that participants understood the procedure. At the end of each experimental block, feedback on overall accuracy (\% correct) and RT (msec) was given.

\section{Data Analysis}

RT for correct responses was our chief measure of interest. Effects were considered significant on the basis of an alpha level of .05 , and post hoc pairwise comparisons were Bonferroni corrected. Mean and maximum error rates, respectively, were $10.5 \% / 11.8 \%$ for "earlier" instruction participants and $10.1 \% / 12.3 \%$ for "later" instruction participants. RT was examined as a function of the serial positions of the earlier- and later-probe items, as outlined at the beginning of this article. The critical test of search direction depended on measuring the serial position slope of the earlier-probe item while

\section{A Earlier Instruction}

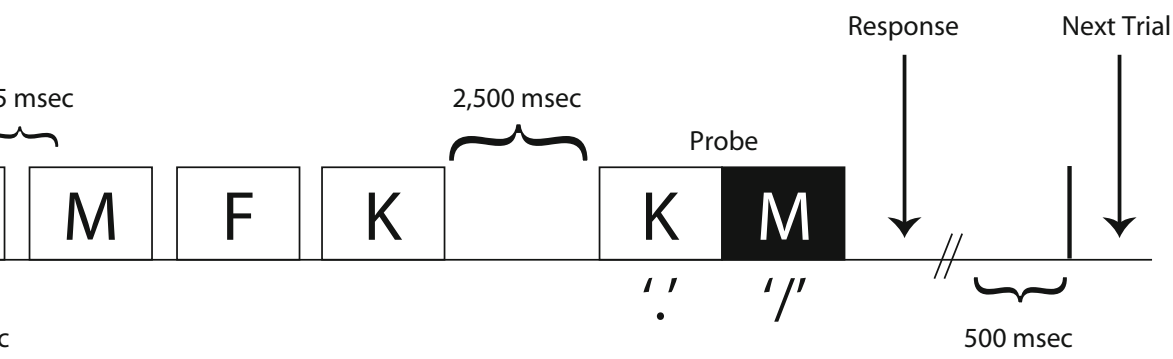

\section{B Later Instruction}

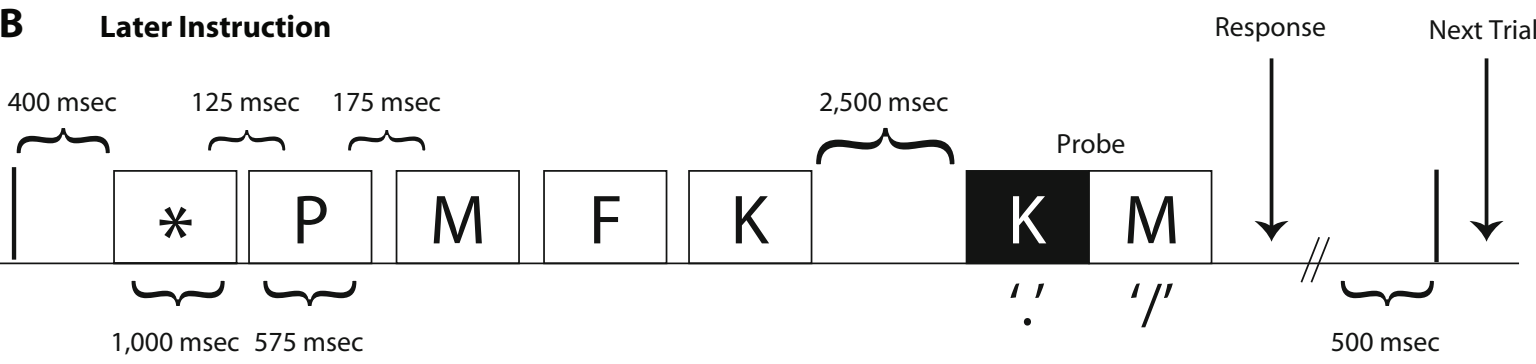

Figure 2. Time course of one experimental trial. Example of the "earlier" and "later" instructions using a four-item list. At test, two items are presented in random order, and the participant is asked to respond to the probe stimulus that occurred earlier ("earlier" instruction) or later ("later" instruction) in the just-presented list. The correct response item is depicted on a dark background in this figure only, not in the experiment itself. The key the participant would use to select each probe item is depicted underneath the probe item itself. 
holding the later-probe item fixed, and measuring the serial position slope of the later-probe item while holding the earlier-probe item fixed. We estimated these slopes with linear regression, resulting in a total of LL-2 slopes for each LL and for each participant. To increase power and facilitate data analysis, we averaged these slopes across probe lags within each LL. Initially, we kept RT data separated for probes that were presented in the intact configuration (earlier-probe item on the left and later-probe item on the right) versus the reversed configuration (earlier-probe item on the right and later-probe item on the left). This resulted in four mean slopes for the earlier-probe position and four for the later-probe position for all combinations of LLs and probe configurations. Repeated measures ANOVAs were performed for the RT slopes for the earlier- and laterprobe items separately with two within-subjects factors, "list length" (four levels: $3,4,5,6$ ) and "probe configuration" (two levels: intact vs. reversed), and one between-subjects factor, "instruction" ("earlier" vs. "later"). Because no main effects of "list length" or "probe configuration" or interactions were significant (all $p \mathrm{~s}>.1$ ), we averaged the serial position slopes across LL and probe configuration. For illustration purposes, we present the RT data as 3-D bar graphs (Figures $1 \mathrm{G}$ and $1 \mathrm{H}$ ), equivalent to the illustrations of our hypotheses in Figures 1A and 1B. When data are collapsed across LLs, each LL will contribute to a different subset of the bars in the 3-D bar graphs. Thus, for the "earlier" instruction, serial positions were measured relative to the start of the list, whereas for the "later" instruction, serial positions were measured relative to the end-of-list (position "LL") item.

\section{RESULTS}

Visual inspection of the observed RT data (Figures $1 \mathrm{G}$ and $1 \mathrm{H}$ ) and derived slope measures, as illustrated in Figures $1 \mathrm{I}$ and $1 \mathrm{~J}$, revealed a qualitative pattern that suggests that the "earlier" instruction data predominantly support the forward self-terminating model. Moreover, the 3-D bar graphs reinforce the resemblance of the "earlier" instruction data (Figure $1 \mathrm{G}$ ) to the forward self-terminating prediction (Figure 1A). Indeed, the slope for the earlierprobe item serial position was significantly larger than zero $[t(39)=7.96, p<.001]$, and the slope for the serial position of the later-probe item was close to zero $[t(39)=1.94$, n.s. $]$, as predicted in Figures $1 \mathrm{C}$ and 1D. In contrast, the "later" instruction data (Figures $1 \mathrm{~K}$ and $1 \mathrm{~L}$ ) predominantly supported the backward self-terminating model (Figures 1E and 1F), with a near zero, nonsignificant slope for the serial position of the earlier-probe item $[t(39)=0.93$, n.s. $]$, but a significantly negative slope for the later-probe item serial position $[t(39)=-6.46, p<$ $.001]$. It is important to note that, although the "earlier" and "later" instructions differed in terms of which scanning direction they resembled most, the RT data did not fit the predictions perfectly; rather, the observed serial position functions appear curvilinear, particularly for the "later" instruction. Although the observed qualitative differences between instructions corroborated our hypothesis, the proposed simple forms of forward and backward self-terminating search cannot fully explain participants' performance in this task.

\section{Overall Performance Measures}

Apart from direction of search's differing between instructions, we asked whether one instruction was less effective than the other by comparing the following overall performance measures: mean error rate, mean RT, and slope of RT as a function of LL, which is thought to be related to the rate of memory scanning (Sternberg, 1967). RT and mean error rate were regressed onto LL for each participant, and slope of RT versus LL and LL zero-intercept were computed for each participant before carrying out statistics across participants. Linear regressions of RT on LL are plotted in Figure 3. A linear model explained about $99 \%$ of the variance in both cases. Independent-samples $t$ tests found no significant differences between "earlier" and "later" instructions in mean error rate, slope, or zero intercept of RT versus LL. Mean RT was higher for the "later" $(M=1,550 \mathrm{msec}, S D=70)$ than for the "earlier" $(M=1,311 \mathrm{msec}, S D=69)$ instruction $[t(78)=10.2, p<$ $.05]$. Error rate and slope of RT versus LL did not differ significantly between instructions. A small but significant overall speed advantage was, therefore, found for the "earlier" instruction.

\section{Scanning Rate Differences Between Instructions}

Finally, we asked whether scanning rate, as measured by the significant serial position slope, differed between instructions. An independent-samples $t$ test on the absolute values of slope found the serial position slope for the laterprobe item in the "later" instruction to be significantly smaller than the serial position slope for the earlier-probe item in the "earlier" instruction $[t(78)=10.2, p<.001$; mean slopes: earlier-probe item in "earlier" $=234 \mathrm{msec} /$ item; later-probe item in "later" $=-170 \mathrm{msec} /$ item].

\section{DISCUSSION}

Our results support the hypothesis that even when the formal judgment is identical, the wording of JOR instructions may induce large qualitative differences in search strategy. Our results suggest a range of effective search strategies in short-list JORs. Specifically, there are at least two effective but distinct search strategies, both selfterminating (Townsend \& Wenger, 2004), that replicate prior findings in subspan JORs (Table 1). However, the strategies differed in search direction, depending on the precise wording of the instructions. Our findings challenge three classes of models, as discussed below.

\section{Instruction Effects on Search Direction}

We found an effect of instruction: The serial position slope in the "earlier" instruction was significantly positive for the position of the earlier-probe item, while showing nonsignificant serial position slope for the position of the later-probe item (Figures $1 \mathrm{G}, 1 \mathrm{I}$, and $1 \mathrm{~J}$ ). This is consistent with the forward self-terminating search schematic in Figures 1A, 1C, and 1D. An opposite pattern was seen in the "later" instruction, where the serial position slope was significantly negative for the position of the later-probe item and nonsignificant for the position of the earlier-probe item (Figures $1 \mathrm{H}, 1 \mathrm{~K}$, and $1 \mathrm{~L}$ ). This is consistent with the backward self-terminating search schematic in Figures 1B, 1E, and 1F. Overall performance measures (Figure 3 ) revealed a significant 

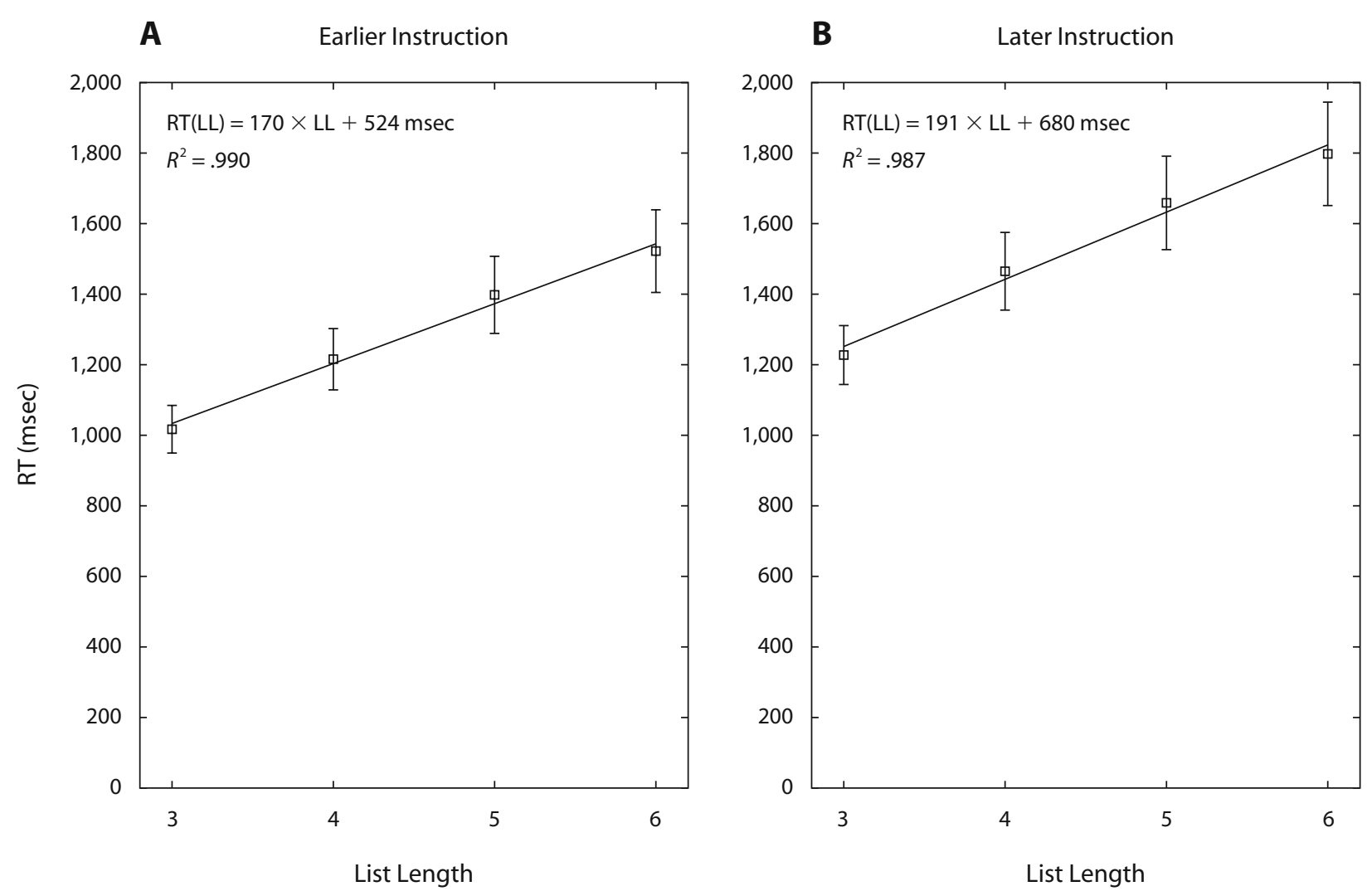

Figure 3. Linear regression for response times (RTs) as a function of list length for each instruction. Error bars denote standard error of the mean across participants.

overall speed advantage for the "earlier" instruction, but the serial position slope was shallower for the "later" instruction, suggesting not that one search strategy is optimal but rather that the strategies reflect different trade-offs.

\section{Participants' Treatment of the Dual-Item Probe}

The JOR involves a two-item probe. It is of interest to know how the two probe items are processed. For example, if the two items were processed one after the other with one probe processed first, there would be a bias toward the left-sided or right-sided probe item during the comparison process. We found no main effect or interactions with probe configuration ("intact" vs. "reversed"), suggesting that neither the left-sided nor the right-sided probe item dominates the memory search process (Hacker, 1980).

\section{Comparison With Prior Findings}

Our results for the "later" instruction are consistent with data from Hacker (1980), McElree and Dosher (1993), and Muter (1979), in that a robust backward, self-terminating search was observed. This suggests that factors in their experiments (Table 1), such as the short retention intervals and material types, were not alone responsible for backward, self-terminating search. Sternberg (1969) reported forward, self-terminating search; however, it was unclear whether the result was a consequence of his unique pro- cedure, which included postpresentation forward serial recall, repeated presentations of the list, and testing exclusively successive probe pairs. We did not include these factors. Our "earlier" instruction replicated the forward scanning pattern, suggesting that forward scanning is not uniquely reliant on Sternberg's (1969) procedure.

\section{Implications for Models}

Our data inform the following four classes of models.

Exhaustive models. In exhaustive search, the memory comparison process continues, regardless of a positive or negative match. Substantial serial position effects were observed for both instructions, representing definitive evidence against purely exhaustive search (Townsend \& Wenger, 2004).

Distinctiveness-based models. In distinctivenessbased models, recall is dependent on how "distinct" items are from one another. In judgments of relative order, performance should be better the greater the distance between the two probe items' positions (Neath \& Crowder, 1996), as found in long lists (e.g., McDaniel \& Bugg, 2008; Yntema \& Trask, 1963). A so-called "distance effect" (Estes, 1972) in our data would consist of a positive slope for the earlier-probe item position, combined with a negative slope for the later-probe item position. Consistent with prior subspan findings, we did not observe distance effects, suggesting that distance effects are not a 
dominant pattern for either of our instructions. This challenges temporal and contextual distinctiveness accounts of judgments of relative order in short, subspan lists.

Limited-capacity parallel models. In limitedcapacity parallel search, the presence of additional items in the search set slows all search rates, enabling the model to explain an increase in RT with increasing LL (Townsend \& Wenger, 2004). Consequently, an LL effect should be observed in scanning rate as measured by the serial position slope. Since this measure did not vary across LL in instructions, limited-capacity parallel models are challenged.

Unlimited-capacity parallel models. The absence of an LL effect in serial position slope is, however, compatible with unlimited-capacity parallel models, wherein the search rate is constant, regardless of LL. Thus, an alternate account of our findings could be made within unlimitedcapacity, parallel-search models. As suggested in the beginning of the article, rather than referring to forward and backward scanning direction (as is appropriate for serial scanning models), one could refer to scanning operation speeds increasing (in analogy to Page \& Norris, 1998) or decreasing as a function of serial position, respectively.

Hybrid/mixture models. More complex model accounts are possible, of course. Whereas serial position effects challenge pure exhaustive models, one could propose, for example, a two-pass model, wherein the participant first searches exhaustively, then performs a selfterminating search. With the present data, we cannot rule out these more complex models.

\section{Conclusion}

Prior studies were inconsistent regarding whether relative order memory search in subspan lists proceeds in the forward or backward direction. Our findings suggest that both strategies are easily induced, and reveal a robust effect of instruction on scanning direction. Inconsistent findings may be reconciled by considering how subtle differences in instructions may orient the participant's attention predominantly toward the start or the end of a list. We observed two effective but distinct memory search strategies: The "earlier" instruction supported the forward-scanning, self-terminating model, whereas the "later" instruction supported the backward-scanning, selfterminating strategy. Absences of (1) an LL effect on serial position slope, (2) distance effects, and (3) flat serial position curves are incompatible with limited-capacity parallel-search, distinctiveness-based, and purely exhaustive models, respectively, for JOR in short, subspan lists. Finally, our findings suggest that the cognitive basis of relative order judgments in memory is more flexible than previously thought.

\section{AUTHOR NOTE}

We thank Chris Madan for assistance in implementing the task, and Saul Sternberg and Bennet Murdock for helpful feedback on the manuscript. Correspondence concerning this article should be addressed to M. Chan, Department of Psychology, University of Alberta, Edmonton, AB, T6G 2E9 Canada (e-mail: mc3@ualberta.ca).

\section{REFERENCES}

EsTES, W. K. (1972). An associative basis for coding and organization in memory. In A. W. Melton \& E. Martin (Eds.), Coding processes in human memory (pp. 161-190). Washington, DC: Winston.

FoZARD, J. L. (1970). Apparent recency of unrelated pictures and nouns presented in the same sequence. Journal of Experimental Psychology, 86, 137-143.

Geller, A. S., Schleifer, I. K., Sederberg, P. B., Jacobs, J., \& Kahana, M. J. (2007). PyEPL: A cross-platform experimentprogramming library. Behavior Research Methods, 39, 950-958.

HACKER, M. J. (1980). Speed and accuracy of recency judgments for events in short-term memory. Journal of Experimental Psychology: Human Learning \& Memory, 6, 651-675.

Jensen, O., \& Lisman, J. E. (1998). An oscillatory short-term memory buffer model can account for data on the Sternberg task. Journal of Neuroscience, 18, 10688-10699.

Kahana, M. J. (1996). Associate retrieval processes in free recall. Memory \& Cognition, 24, 103-109.

Kesner, R. P., Hopkins, R. O., \& Fineman, B. (1994). Item and order dissociation in humans with prefrontal cortex damage. Neuropsychologia, 32, 881-891.

MCDANIEL, M. A., \& BUGG, J. M. (2008). Instability in memory phenomena: A common puzzle and a unifying explanation. Psychonomic Bulletin \& Review, 15, 237-255.

McElree, B., \& Dosher, B. A. (1993). Serial retrieval processes in the recovery of order information. Journal of Experimental Psychology: General, 122, 291-315.

Murdock, B. B. (1974). Human memory: Theory and data. Oxford: Erlbaum.

Murdock, B. B. (1982). A theory for the storage and retrieval of item and associative information. Psychological Review, 89, 609-626.

Muter, P. (1979). Response latencies in discriminations of recency. Journal of Experimental Psychology: Human Learning \& Memory, 5, 160-169.

Neath, I., \& Crowder, R. G. (1996). Distinctiveness and very shortterm serial position effects. Memory, 4, 225-242.

Page, M. P., \& NorRIs, D. (1998). The primacy model: A new model of immediate serial recall. Psychological Review, 105, 761-781.

STERNBERG, S. (1967). Retrieval of contextual information from memory. Psychonomic Science, 8, 55-56.

SternBerg, S. (1969). Memory-scanning: Mental processes revealed by reaction-time experiments. American Scientist, 57, 421-457.

Townsend, J. T., \& Wenger, M. J. (2004). The serial-parallel dilemma: A case study in a linkage of theory and method. Psychonomic Bulletin \& Review, 11, 391-418.

van Vugt, M. K., Schulze-Bonhage, A., Litt, B., Brandt, A., \& Kahana, M. J. (2009). Hippocampal gamma oscillations increase with working memory load. Manuscript submitted for publication.

WolfF, P. (1966). Trace quality in the temporal ordering of events. Perceptual \& Motor Skills, 22, 283-286.

Yntema, D. B., \& TRAsK, F. P. (1963). Recall as a search process. Journal of Verbal Learning \& Verbal Behavior, 2, 65-74.

(Manuscript received October 3, 2008; revision accepted for publication June 12, 2009.) 